\title{
'It's not a romantic relationship': Stories of Adoption and Abandonment of Smart Speakers at Home
}

\author{
Alexandra Voit \\ VIS, University of Stuttgart \\ Stuttgart, Germany \\ info@alexandra-voit.de \\ Maike Ernst \\ VIS, University of Stuttgart \\ Stuttgart, Germany \\ ernst.maike7@gmail.com
}

\author{
Jasmin Niess \\ University of Bremen \\ Bremen, Germany \\ niessj@uni-bremen.de
Henrike Weingärtner
VIS, University of Stuttgart \\ Stuttgart, Germany \\ weingahe@fius.informatik.uni- \\ stuttgart.de
}

\author{
Caroline Eckerth \\ LMU Munich \\ Munich, Germany \\ caroline.eckerth@gmx.de \\ Paweł W. Woźniak \\ Utrecht University \\ Utrecht, the Netherlands \\ p.w.wozniak@uu.nl
}

\begin{abstract}
Smart speakers become increasingly ubiquitous in our homes. Consequently, we need to study how smart speakers affect the members of a household. Understanding the adoption of a smart speaker can assure it does not negatively influence the social dynamics within a household and create opportunities for further assistance. We deployed an Amazon Echo dot in nine households with 20 inhabitants who were new smart speaker users. We conducted multiple interviews, inquiring how a smart speaker was integrated into a household from day one. We investigated the development of social rules around using the device and how the smart speaker was appropriated. Users developed different strategies of using the device which altered social behaviours in some households. Further, we identified barriers and unmet requirements in introducing smart speakers to home environments. Our work contributes to an understanding of ubiquitous assistance for user groups at home.
\end{abstract}

\section{CCS CONCEPTS}

- Human-centered computing $\rightarrow$ Human computer interaction (HCI); Field studies; Empirical studies in ubiquitous and mobile computing.

\section{KEYWORDS}

Smart Speaker, Voice Assistants, Voice I/O, Smart Home

\section{ACM Reference Format:}

Alexandra Voit, Jasmin Niess, Caroline Eckerth, Maike Ernst, Henrike Weingärtner, and Paweł W. Woźniak. 2020. 'It's not a romantic relationship' Stories of Adoption and Abandonment of Smart Speakers at Home. In 19th International Conference on Mobile and Ubiquitous Multimedia (MUM 2020),

Permission to make digital or hard copies of all or part of this work for personal or classroom use is granted without fee provided that copies are not made or distributed for profit or commercial advantage and that copies bear this notice and the full citation on the first page. Copyrights for components of this work owned by others than the author(s) must be honored. Abstracting with credit is permitted. To copy otherwise, or republish, to post on servers or to redistribute to lists, requires prior specific permission and/or a fee. Request permissions from permissions@acm.org.

MUM 2020, November 22-25, 2020, Essen, Germany

(c) 2020 Copyright held by the owner/author(s). Publication rights licensed to ACM.

ACM ISBN 978-1-4503-8870-2/20/11...\$

https://doi.org/10.1145/3428361.3428469

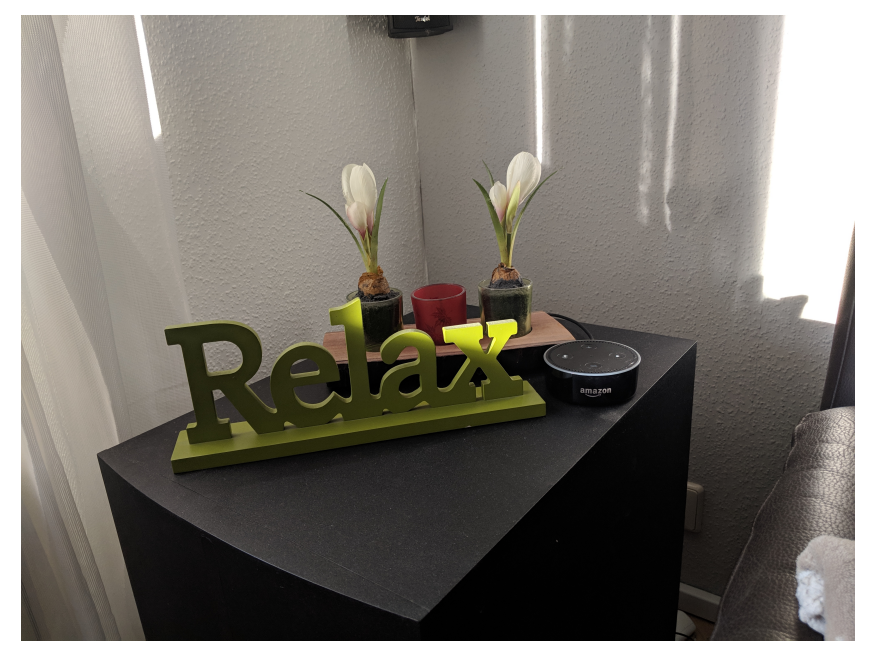

Figure 1: Example for a deployed Amazon Echo Dot, placed in the living room of a participant's home.

November 22-25, 2020, Essen, Germany. ACM, New York, NY, USA, 12 pages. https://doi.org/10.1145/3428361.3428469

\section{INTRODUCTION}

Nowadays, smart speakers are rapidly becoming everyday devices and are steadily permeating everyday environments. More than 146 million smart speakers were sold in 2019 alone $^{1}$. As these devices enter our everyday lives and offer new functionalities, they become part of the everyday experience. All smart speakers feature a conversational interface, a new conversation partner, which is designed for regular 'communication' with the user. As a consequence, understanding the everyday experience of smart speakers and how they augment and alter our interactions with other devices and people becomes a relevant question for Human-Computer Interaction (HCI).

The HCI field has a history of researching smart speakers since they first became available to a wide audience. Studies addressed establishing habits when using smart speakers [3] or how smart speakers provide a new dimension to at-home accessibility [41]. To

${ }^{1}$ https://techcrunch.com/2020/02/17/smart-speaker-sales-reached-new-record-of146-9m-in-2019-up-70-from-2018/ 
date, less attention was given to a smart speaker's initial experience after it is acquired and enters the home space for the first time. As smart speakers become more affordable to a broader range of users, it is important from a societal point of view to understand the first days of using a smart speaker at home. This way, research can ensure that smart speakers provide users with new functionalities and empower them to complete home tasks more efficiently, while not disrupting existing routines or social habits at home. Further, as smart speakers enter the households, they encounter different social configurations with the home.

To explore how smart speakers are integrated into households and what the social implications of their usage are, we conducted a field study. We introduced 20 participants in differently organized households to the Amazon Echo Dot (see Figure 1). We asked participants to use the device for four weeks and conducted initial, mid-term, and final interviews to explore how the users integrated and experienced the smart speakers in their daily lives. Further, we investigated if the users developed a specific attitude and/or attachment to the smart speaker over time. Similar to the previous work [4], we investigated the experience of first-time smart speaker users. Hereby, we focused on how users living in different social settings integrate smart speakers into their daily lives. Finally, we placed particular emphasis on the emergence (or not) of new or changing social behaviors connected to the presence of smart speakers in the household. We investigated both how human relationships change with the presence of the speaker and how the smart speaker became a social actor.

Using qualitative analysis, we identified two user groups of smart speakers. In half of the households, smart speaker usage steadily faded. Users reported that the devices failed in fitting into the life of the house. In the other half of the studied homes, users anthropomorphized the device and quickly formed habits related to the Echo Dot. In this paper, we look into details of a smart speaker's (non-) adoption process by describing the following themes: CONCERNS AND Nuisances, Establishing Habits, Building RAPPORT, and Relationship Formation Over Time.

This paper contributes the following; (1) A four-week qualitative study of how 20 participants experienced their first smart speaker; (2) four themes that characterize how smart speakers are introduced to home environments, the process of the (non-) adoption of the device and the social behaviors connected to the Echo Dot; and (3) implications for the design of future smart speakers that provide an improved experience and better support social dynamics at home. These implications can be used to guide the development of the next generation of smart speakers.

\section{BACKGROUND AND RELATED WORK}

Here, we report on the work that informed and inspired our inquiry. First, we review recent research on understanding the experience of using conversational agents and especially smart speakers. Then, we explore past reports on how users develop relationships with technologies over time or abandon them and the methods of studying technologies in home environments.

\subsection{Conversational Agents \& Smart Speakers}

Former work that studied conversational agents revealed that the majority of the users was not able to accurately judge the system capacities of conversational agents. Especially, users without knowledge of computer science were missing a clear mental model of how conversational agents work [27]. That resulted in a tendency to anthropomorphize the conversational agent and setting unrealistic expectations.

A body of work investigated how users interact with conversational agents $[6,8,9,15,29,40]$. Clark et al. investigated differences between human-to-human and human-to-agent conversations. They found that participants used similar interlocutor characteristics in communication with strangers or casual acquaintances and communication with conversational agents [6]. However, their participants questioned the need for bonding and developing a relationship with conversational agents. Further, Porcheron et al. showed that users predominantly react to failures of conversational agents by repeating the original query or reformulating it [40].

Other work identified barriers that act as barriers for users of conversational agents [9]. Users were often frustrated by the need to combine touch and speech interaction to interact to conversational agents, e.g., selecting a contact to call or unlocking the phone before a query can be entered [9]. Further, users prefer to enter non-private data to conversational agents $[15,29]$ and to use conversational agents in safe, domestic environments [29]. Reported reasons for avoiding speech interaction in public were mainly privacy concerns [29], embarrassment in front of strangers [8,29] and cultural factors [8].

Research that addresses smart speakers is inherently related to understanding conversational agents. In contrast to conversational agents on mobile phones or laptops, smart speakers are placed at a static position in the users' homes [46]. Smart speakers have an integrated virtual assistant that can be activated through an activation word. For instance, Amazon Echo, the smart speaker from Amazon connects to the voice-controlled intelligent personal assistance service Alexa. However, as most participants in studies use the terms Alexa, Echo and smart speaker interchangeably [42], this paper treats them as synonymous with the service that the Amazon smart speaker provides.

Several recent works studied different aspects of how users interact with smart speakers [1, 3, 39]. Bentley et al. [3] investigated habits in smart speaker use through analyzing an extensive database of voice history logs from Google Home. They found that playing music was by far the most used action on the smart speaker. As the users owned the device longer, music usage was still high, but it declined. On the other hand, users used more automation increasingly, suggesting that the smart speaker was further integrated into the home environment. Porcheron et al. [39] analyzed audio data from a month-long Amazon Echo usage period to study the intricacies of the dialogues between users and smart speakers. They found that the 'atomic' way one communicated with a smart speaker bore little resemblance to real conversations. Beneteau et al. analyzed situation in which the communication between family members and the smart speaker was breaking down and found that in these cases family members often collaborate to repair the communication to the smart speaker by discoursing scaffolding and 
varying the speech, e.g., the pronunciation, and language, e.g., the used wording [1]. Sciuto et al. [46] studied usage logs and conducted interviews with users of Amazon Alexa. They found that users of smart speakers explored the functionality of smart speakers within the first few days. Afterwards, their participants used the smart speaker constantly week-over-week for the first year. However, the amount of daily requests varied between the households.

Other work studied how users experience smart speakers [24, 41] or investigated privacy perceptions $[28,30]$. Lau et al. conducted a diary studies and interviews with smart speaker users and interviews with non-users of smart speakers about their experience while focusing on privacy perceptions [24]. They found that nonusers do not see utility in smart speakers, while users users of smart speakers are aware that they trade in their privacy for convenience. Users as well as non-users of smart speakers are distrusting the speaker companies. Manikonda et al. showed that users of smart speakers prefer to use them in their daily lives although they are concerned about privacy, e.g., about being hacked or about the data collection and data storage [28]. Similarly, Benlian et al. reported that conversational agents can with their anthropomorphic design features can weaken the influence of privacy issues [2]. Moorthy and $\mathrm{Vu}$ analyzed privacy and security issues that are caused by conversational agents [30]. They showed by investigating different possible attacks that the interaction of the users with the conversational agents are the weakest link. Here, one of the reasons is the used (predefined) wake word of the conversational agents that are easy to guess by others and can also be triggered by external sources such advertisements shown on the TV. Tabassum et al. identified the trust paradox regarding smart home appliances such as smart speakers [49] Although their participants were aware of security issues caused by the storage in the cloud as well as the data sharing, their participants also trusted that the manufactures will not misuse the data because of possible outcomes of a data misuse such as loosing the reputation. Pradhan et al. [41] analyzed Amazon reviews and interviews to study how smart speakers supported accessibility. They found that smart speakers at home were perceived as particularly beneficial by participants with a vision impairment who used the devices actively to facilitate many everyday actions.

\subsection{Designing for the home}

Designing and exploring interactive systems for the home has a long tradition in HCI research. Almost two decades ago, Edwards and Grinter [16] identified seven challenges in the context of ubiquitous computing for the home. For instance, one challenge the authors discussed was 'Designing for Domestic Use'. They emphasized the need for designers to build an in-depth understanding of the complex routines of the home and how these might lead to the adoption or the abandonment of new technologies. We aim to shed more light on these processes and their consequences. Further, Grinter and Edwards investigated how households make the home network works and found that households do not only need to manage the coordination of the usage of different home appliances but also the effort to configure and manage the appliances [22]. Further, Tolmie et al. found that designers for networking technologies in domestic environments need to take the existing infrastructure in the home into account and need to consider how future technologies can be integrated into existing routines [50].

Pyae and Joelsson [43] identified seven challenges smart speakers face when they are integrated into the home. Furthermore, Crabtree et al. identified places in the home (i.e., ecological habitats, action, and coordinate displays) such as tables or notice boards that habitually draw the users' attention [10]. These places are prime sites for ubiquitous computing applications that support everyday activities and focus on sharing communication media between the residents in a home context.

Nagel et al. [31] developed and evaluated a light-weight audio communication system for families in their home. This contextaware system supported the application of social norms (e.g., contacting someone in an appropriate moment) and demonstrated how ubiquitous technology can support everyday activities. Oleksik et al. [37] explored the soundscapes in domestic settings. They found that the domestic soundscape is inherently social and creates a need to negotiate social norms. In another vein, Crabtree and Tolmie [12] showcased in their observational study that the assemblage and the arrangement of things are connected to the routines of everyday life. Earlier, Odom et al. [36] investigated how the arrangement and presence of future technologies in teenager bedrooms might affect self-exploration and identity construction processes. These works show how interactive systems are easily integrated into everyday routines and have a potentially transformative character.

\subsection{Abandonment and non-use}

Some studies of smart speakers reported that participants reduced their interactions over time [46], whereas other results indicate that there is no decline regarding the usage [3]. However, to the best of our knowledge, to date, only two studies $[5,20]$ have focused explicitly on the abandonment of a class of devices that included smart speakers. Cho et al. investigated the abandonment of smart speakers in a long-term diary study with first-time users of smart speakers [5]. They found that the reasons for the abandonment of smart speakers after a few weeks of usage are according to a disappointing exploration leading to minimal usage or abandonment. Garg and Kim [20] conducted an exploratory study to build an understanding of the usage of the Internet of Things (e.g., voice assistants, smart watches, smart locks). Their preliminary results showed that participants mainly stopped using devices due to demotivating interactions (e.g., distracting notifications, notifications of failure to achieve a goal). Further, they found that participants stopped using the device when it was too complicated to use or provided unnecessary, confusing information. Only a few participants mentioned privacy concerns as a determining factor regarding continued usage. One of the relevant factors for continuing usage was autonomy in daily activities.

Other work investigated the reasons for non-usage of of devices [45][44]. Satchell et al. found that the adoption of the technology could be lagging, active resistance by the users, disenchantment, disenfranchisement, displacement and disinterest [45]. Sambasivan et al. identified the reasons avoidance by the user (e.g., by turning the devices off), pretending usage by the user, and resistance to devices that were forced on them [44]. 


\subsection{Anthropomorphism of digital assistants}

There is an increasing interest in the HCI community to explore the intricacies of designing future digital assistant technologies [7]. However, it still remains a challenge to design technologies that can become meaningful digital companions [34], i.e., technologies that carry personal and social meaning. Lopatovska and Williams [26] reported that a significant share of Amazon Echo's users expected the device to exhibit social behavior. Preliminary results by Purington et al. [42] suggest that users tend to ascribe human qualities to smart speakers despite the 'non-human' conversation style. They analyzed user reviews of the Amazon Echo and found that user satisfaction seems to be connected to more personification of the technology.

The class of behaviors mentioned above can be called anthropomorphism and defined as the attribution of human-like characteristics, motivations, emotions or intentions to non-human agents, such as animals or objects [17]. Epley et al. [17] state that anthropomorphizing serves three purposes, namely: (1) making sense of situations, (2) reducing uncertainty in specific situations, and (3) establishing social connections. Early research in $\mathrm{HCI}$ found that humans react towards technologies in social ways [32, 33]. However, Nass and colleagues take another, contrasting stance compared to Epley et al. and state that these social reactions are triggered by social cues [33]. Kuzminykh observed that the antropomorphization of smart speakers is related to their implemented behavior regarding the categories approachability, sentiment, professionalism, intelligence, and individuality, e.g., Alexa is perceived as genuine and caring while Siri is perceived as cunning and disingenuous [23] Further, Gao et al. found that users who are anthropomorphizing smart speakers are also showing more positive emotions than users who treated the smart speaker as an electronic device [19].

\subsection{Summary}

Past work about conversational agents and smart speakers shows that the interaction opportunities offered by these devices are often limited once they are deployed in the real world. This research inspires our inquiry to study first-time users, the evolution of their initial relationship with a new conversational agent at home as well as whether the presence of smart speakers could influence social dynamics.

Our work is interestingly different from past research by former work about adoption [42] or abandonment [5,20] as it specifically explores how smart speakers affect social interactions in households and how the use of smart speakers is affected by the social environment. Further, it is still not fully understood what relationships users form with smart speakers (if any) and how that process is enacted. Our work aims to shed more light on this issue. This way, we contribute a broader understanding of the adoption or abandonment of smart speakers in households with different social settings and how they can better support social dynamics in the home.

\section{METHOD}

To gain an understanding of how users integrate smart speakers when they are introduced to their home environments and how they experience smart speakers in their daily lives, we conducted a four-week in-situ study where we deployed the Amazon Echo Dot in a variety of households.

\subsection{Procedure}

At the beginning of the study, we visited all participants in their homes. First, we asked all household members (except underage children) to sign the consent form and answer the demographic questionnaire. Afterward, we conducted an initial semi-structured interview with each household member individually (except underage children). At the end of our first visit, we asked the participants where they would like to place the Amazon Echo Dot (Version 2) in their homes. However, we required that the Amazon Echo Dot were placed in a shared location in their homes (i.e., kitchen, living room) to ensure access to the device for all household members. In addition to the device, we provided the official Amazon Echo Dot's guidance sheet instead of showing the participants the functions of the smart speaker to ensure comparability with regular users of smart speakers. After we deployed the Amazon Echo Dot, we asked the participants to use the device for the next four weeks. We have not further encouraged the participants to interact with the smart speakers to avoid to influence their behaviour. The study started on the day after deploying the Echo Dot. Two weeks after the initial deployment, we revisited the participants in their homes and conducted the mid-term semi-structured interviews with every household member individually. At the end of the study (i.e., after four weeks), we visited the participants, conducted the final semi-structured interview with each participant individually, and collected the deployed Amazon Echo Dots. Finally, we rewarded every household with a EUR 40 Amazon voucher for their participation in the study.

\subsection{Participants}

In the participant selection process, we explicitly looked for households with diverse living situations to be able to investigate the integration of smart speakers in different social settings. Therefore, we recruited households with different settings including people living alone, couples and families living together as well as people living in shared apartments. Another requirement for the participation in our study was that the participants could not have used a smart speaker before, but were willing to test and use a smart speaker for the study duration (i.e., four weeks). In total, nine households with 20 household members (including three children aged between 1 and 6; $M=3.66, S D=2.52$ ) living in Germany participated in the study. Since all children were underage and could not give consent, we excluded all children from the interviews. The remaining 17 adult participants ( 5 female, 12 male) were aged between 21 and 35 years $(M=26.29, S D=4.18$; cf. Table 1$)$. Five households placed the smart speaker in the kitchen; three in the living room, one participant, placed the speaker in his single-room apartment. Two households were single-person households, and three households were shared apartments. Further, two couples and two families with their underage children participated in our study. All participants owned a smartphone, and all but one participant also owned a laptop/desktop computer. Further, one household owned smart lights. In total, eight participants stated that they had previously used conversational agents integrated to their smartphones, i.e., Siri or 
Table 1: Overview of the participants. The first digit of the participant id (PID) encodes the household the participant resided in. We use the notation $P \# X$ to refer to the entire household, e.g. P1X refers to P11 and P12 collectively.

\begin{tabular}{|c|c|c|c|c|c|}
\hline & PID & Age & Gender & Highest Education level & Occupation \\
\hline \multirow{7}{*}{ 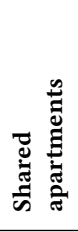 } & P11 & 23 & $\mathrm{~m}$ & High School & Student \\
\hline & P12 & 27 & $\mathrm{~m}$ & High School & Student \\
\hline & P31 & 21 & $\mathrm{~m}$ & High School & Student \\
\hline & P32 & 22 & $\mathrm{~m}$ & High School & Student \\
\hline & P33 & 23 & $\mathrm{~m}$ & High School & Student \\
\hline & P71 & 35 & $\mathrm{~m}$ & Bachelor & Employee \\
\hline & P72 & 28 & $\mathrm{~m}$ & Bachelor & Employee \\
\hline \multirow{7}{*}{ 全 } & P81 & 34 & $\mathrm{f}$ & Vocational training & Employee \\
\hline & P82 & 38 & $\mathrm{~m}$ & Vocational training & Employee \\
\hline & P83 & 6 & $\mathrm{~m}$ & - & - \\
\hline & P84 & 4 & $\mathrm{~m}$ & - & - \\
\hline & P91 & 28 & $\mathrm{f}$ & Vocational training & Employee \\
\hline & P92 & 25 & $\mathrm{~m}$ & Bachelor & Employee \\
\hline & P93 & 1 & $\mathrm{~m}$ & - & - \\
\hline \multirow{4}{*}{$\frac{8}{3}$} & $\mathrm{P} 21$ & 23 & $\mathrm{~m}$ & High School & Student \\
\hline & $\mathrm{P} 22$ & 22 & $\mathrm{f}$ & High School & Student \\
\hline & P41 & 26 & $\mathrm{~m}$ & Bachelor & Employee \\
\hline & $\mathrm{P} 42$ & 25 & $\mathrm{f}$ & Vocational training & Employee \\
\hline \multirow{2}{*}{ 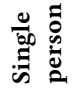 } & P51 & 32 & $\mathrm{f}$ & Bachelor & Employee \\
\hline & P61 & 25 & $\mathrm{~m}$ & High School & Student \\
\hline
\end{tabular}

the Google Assistant, although seven participants mentioned that they were using it infrequently. All participants stated that they were not using conversational agents integrated into their laptops or desktop computers.

\subsection{Interviews}

In total, we conducted three interviews individually with each household member. We opted to interview participants individually in order to capture their unique perception of how the smart speaker affected the household. We informed them that their opinions would stay confidential and anonymized which enabled sharing information that could be viewed negatively by other members of the household.

Initial interview: In the initial interviews, we asked the participants about their experiences with voice assistant systems, their knowledge about smart speakers, and the functionalities provided by Amazon Alexa. Further, we investigated how they usually accomplished daily tasks and activities that could be supported by the Amazon Echo Dot, such as playing music or checking weather conditions [3].

Mid-term interview: In the mid-term interviews, we investigated how the participants used the Amazon Echo Dot in their daily lives so far (i.e., how often and why they interacted with Amazon Echo Dot, which functions or additional skills they explored). Further, we investigated if technical support was needed.

Final interview: In the final interviews, we asked the participants to reflect on their usage behavior and if they would use a smart speaker system in their homes in the future. We aimed to elicit stories of using Amazon Echo Dot in their daily lives. We were especially interested in how the participants integrated the Amazon Echo Dot into their household and if they changed their usual routines. We also addressed changes in usage over time. We used interpretive questioning [14] to gain more information about concepts of particular importance to our study once they were mentioned in the stories. The interview script paid specific attention to instances where participants discussed their relationship to the Amazon Echo Dot, possible anthropomorphism of the devices and differences in the interaction with the device when other household members or visitors were present.

\subsection{Data Analysis}

All audio recordings were transcribed verbatim and imported into the Atlas.ti analysis software. Two researchers coded a representative sample of $15 \%$ of the material using thematic analysis with open coding [4]. Next, a coding tree was established through iterative discussion. The remaining transcripts were coded individually by one researcher. A final discussion session was conducted to finalize the coding tree after the material was coded. Three researchers then identified themes in the coded data.

\section{RESULTS}

In general, the user stories reported in our study can be classified into two meta-categories. In four households (P2X, P3X, P8X, P9X), smart speaker usage gradually declined, and the participants hardly ever used the Echo at the end of the study period. In contrast, five households (P1X, P41, P51, P61, P7X) reported using the device steadily throughout the study and developed a relationship to the device. This suggests that the data gathered in the study showcases a spectrum of attitudes towards a smart speaker. The themes, which we identified in the data, often present contrasting attitudes of the participants. In the following, we describe the participants' experiences with the smart speaker in detail through the themes derived from the data, illustrating them with interview quotes. All excerpts were translated from German to English. We begin with the more elementary themes and outline the more complex themes at the end of the results section. An overview about all themes can be found in Table 2 .

\subsection{Concerns and Nuisances}

This theme describes the negative presuppositions and functionality problems the participants expressed during the study and their feelings about the smart speaker as not being helpful or even annoying for subjects. The relatively high error rate during daily use was one of the primary reasons for subjects deciding against using smart speaker more extensively. Furthermore, our subjects feared a violation of their privacy, also about personal data that could be shared with secondary sources, personalized advertisement, and a growing dependence on the smart speaker company that owed their data: "I know, that it is actually stupid when you are doing a lot on the web or when you are active on Facebook [...]. I know, that everything is being tracked anyways, but it may be a kind of psychological component, because you feel screened, because you say to oneself: 'Ok, there is something that is listening permanently'. Because, of course, it 
Table 2: Overview of the identified themes with explanations and examples.

\begin{tabular}{|c|c|c|}
\hline Theme & Short Description & Example \\
\hline $\begin{array}{l}\text { CONCERNS AND } \\
\text { NUISANCES }\end{array}$ & $\begin{array}{l}\text { This theme describes a variety of reasons to not use the } \\
\text { smart speaker in the long run. }\end{array}$ & $\begin{array}{l}\text { Being afraid to lose decision-making } \\
\text { power in purchasing decisions. }\end{array}$ \\
\hline $\begin{array}{l}\text { ESTABLISHING } \\
\text { HABITS }\end{array}$ & $\begin{array}{l}\text { This theme describes the formation of habits in } \\
\text { connection with the smart speaker. }\end{array}$ & $\begin{array}{l}\text { Greeting the smart speaker every } \\
\text { morning. }\end{array}$ \\
\hline $\begin{array}{l}\text { BUILDING RAP- } \\
\text { PORT }\end{array}$ & $\begin{array}{l}\text { This theme describes contrasting insights into the } \\
\text { antropomorphization of the smart speaker. }\end{array}$ & $\begin{array}{l}\text { Feeling less lonely while interacting } \\
\text { with the smart speaker. }\end{array}$ \\
\hline $\begin{array}{l}\text { RELATIONSHIP } \\
\text { FORMATION } \\
\text { OVER TIME }\end{array}$ & $\begin{array}{l}\text { This theme describes how the relationship with the } \\
\text { smart speaker evolved over time and discusses the com- } \\
\text { plexities of the relationship formation in connection } \\
\text { with the different social settings of the participants. }\end{array}$ & $\begin{array}{l}\text { Integrating the smart speaker as an } \\
\text { additional roommate into a shared flat. }\end{array}$ \\
\hline
\end{tabular}

has to listen to you in order to be responsive [...]. Even though it does not make sense at all, but it is just a feeling." (P41, initial interview)

Subjects reported a lack of transparency regarding the use of data by the smart speaker company. Instead, subjects wished to take control of their personal data. It remained unclear to them what exactly happened with their data. Another aspect of concern was the fear buying items through voice interaction unintentionally, or other persons taking advantage of their smart speaker account and buy items with just one voice command. "I would not connect the [Amazon Echo Dot] to my Amazon account. [...] If someone else enters and says "Alexa, buy this and that." [...] However, that's my account. I just do not have any [control] anymore." (P32, mid-term interview)

Some participants were dissatisfied with being the only people in their social group with a smart speaker. They were unable to rely on the opinion and advice of friends and family in using the device. One participant attributed different patterns in smart speaker use to generational issues: "I just believe that the most people who would buy smart speakers [nowadays] are [non-digital-natives, i.e.,] they haven't grown up with a smartphone, and consider it as strange to talk to [smart speakers]. I believe that in the next ten years [people] who would buy smart speakers have grown up with their mobile phones [and that] will change [a lot]." (P12, final interview)

A different household faced the problem of connecting between the functions within the smart speaker. For instance, when the subjects turned off the power of their smart speaker, they were not able to turn on the room lights. They, therefore, became dependent on their smart speaker in order to benefit from their other smart home devices. "Yes, she [Echo] was often turned off. And then, she was turned off again and I wanted to turn on the light, [but that] wasn't working. I had to connect her [before] I was be able to turn the light on. [That] wasn't occurring on a daily basis but happened [every] now and then." (P81, final interview)

Also, in order to make full use of a smart speaker, other smart devices or accounts were required which not all of the users had in advance, which limited their spectrum of use. "In order to be able to use [a smart speaker] appropriately, [I] just need to own a lot of [smart devices such as] smart lights and shutters, that inter-operate with it." (P22, final interview)

\subsection{Establishing Habits}

The second theme describes the ways users establish habits around the smart speaker. In general, users in single households commented more extensively about forming habits around the Echo compared to participants in shared households. One participant described how previously formed habits affected her interaction with technology. She reflected that she did not use it for tasks where she was used to using her phone. Instead, she built new routines around the anthropomorphized smart speaker: "Out of habit, I played music or used the timer of my phone. However, at some point, I established that I'm wishing her a 'Good Morning' every day." (P51, final interview)

In contrast, some participants have been more critical about social interactions with the Echo. One participant described a feeling of unease when there was a possibility of being overheard by someone while talking to the smart speaker. He further reflected about the potential impact these human-technology interactions could have on humanity: P: "Ehm, I feel stupid [when I interact with my echo]. It goes so far that, when I have the window open, I hope that nobody hears me when I speak with my echo, because I feel absolutely stupid."

I: "Can you explain why that is?"

P: "No, not really. It just feels like talking to a device is the next level of distancing oneself from humanity." (P61, final interview)

On a similar note, another participant also preferred to talk to Alexa when nobody could hear the conversation. However, in contrast to the statement above, this was not explained by a critical stance regarding the digitized world. Instead, some participants emphasized that talking to an inanimate artifact made them feel lonely: "I think that's better than talking to her when no one is around. [...] Because somehow it feels strange. [...] On my own, I feel a little stupid talking with such a device. It makes me feel so alone.' (P12, final interview)

Many users mentioned the conscious decision to adjust interactions with the smart speaker by assessing if it was appropriate at the present moment. One participant described a situation, where Alexa was not used in the company of other people to not interrupt their conversation: "I've often used Alexa to turn the music up or down via voice control. But when I had company, I was more likely to operate her with the buttons, because I talked to the person who was 
there at the moment. So I'd rather talk to the human than to Alexa and operated her with the buttons." (P71, final interview)

\subsection{Building Rapport}

This theme describes the development of 'an understanding' around smart speakers and subtle differences in the anthropomorphization of such technologies. We identified two subthemes, one where the participants humanized the technology, independently of their functionality and one where the relationship towards the technology seems to be connected to its functionality.

The participants developed ways to manage situations where the smart speaker did not work as expected, humanizing the device. This is reflected in the following statement, where a participant explained that Alexa was reprimanded if she did not work as expected: "[...] so for me, it's like she's not there sometimes, then I just reprimand her [i.e., Alexa] for being active while she's not supposed to." (P82, mid-term interview)

The following statement illustrates the tendency of some participants to anthropomorphize their device, irrespective of if the smart speaker operated as desired, e.g., activating the speaker when not desired. However, two participants decided to solve the problem proactively. They agreed on calling the technology 'Alexa' when talking about her and 'Echo' when talking to her, in order to make sure that she (i.e., the smart speaker) did not misinterpret the communication: "Oh well, when you talk about Alexa, sometimes she interprets that as if you're talking to her. [...] We compromised by calling her Echo instead." (P11, mid-term interview)

However, some participants had a more practical attitude towards their Echo. One participant stated that the name did not make the smart speaker more human and it did not lead to him appreciating the device more: "My personal relationship with Alexa is pragmatic, not emotional. [...] If the device had a different name, I would call it that. [...] Just because Alexa is called Alexa, it is not human. [...] I know it is a device and nothing more. I haven't developed a personal connection to the device and I would even say that I value my phone ten times more than Alexa." (P41, final interview)

Some participants were unhappy and frustrated about the functionality problems of the smart speaker. One participant reflected that, due to technical issues and because he did not use the Echo regularly, no personal relationship to the technology could form. Interestingly, even though the participant was dissatisfied with the smart speaker the propensity to humanise the technology was also showcased here: "So I think it's like a person you cannot talk to and who upsets you at some point. And you just think 'just be quiet, I do not want to keep talking to you'. [...] We didn't have the slightest personal relationship [...] because I hardly used it and because it did not always work properly. Then you were annoyed again." (P31, final interview)

Interacting with the smart speaker because the fact of using the technology per se was perceived as pleasant was a recurrent topic. Some participants, especially those from single households, felt that the presence and the interactions with Echo made them feel good and less lonely. One participant emphasized how pleasant the voice of Alexa was and reflected about how the interactions with her made him feel: "It's nice to not be so lonely. [...] That's a very pleasant voice. And if someone speaks to you or reads the news to you, it is really... So you are completely alone in an 80-square meter flat, and someone reads the news to you, that has feel-good character. Well, I think that's psychological. It's kind of nice. Apart from that, I don't know. I'm not really a radio listener, but it was great to come to the kitchen and say, 'Alexa play some music', and she just turned on some radio station." (P72, final interview)

\subsection{Relationship Formation Over Time}

This theme describes the intricacies of the social dynamics and the connected relationship formation with the Echo over time. The interactions with Alexa can be situated on a spectrum that ranges from "the speaker" mediating social interactions between different members of a household to the artefact taking on an active role within the social interactions. The social influence of Alexa on individual users can be described as more additive, with some participants building new habits around the anthropomorphized speaker, whereas others maintained a pragmatic relationship to it. In addition, our results reveal an interesting difference in Alexa relationship formation between users with initial privacy concerns and users without initial privacy concerns. Surprisingly, we found that participants who were initially cautious anthropomorphized the smart speaker, despite their initial attitude. However, our findings also show that, potentially due to the reservations towards the technology, the relationship between users with privacy concerns and Alexa remained distant. Hence, we hypothesize that this could be explained by users keeping Alexa at a distance since they doubt the technologies' trustworthiness. The statement from P11 showcases this contrast. The participant described the relationship with Alexa as impersonal. Simultaneously he compared it to a polite, distant human relationship: "My relationship with Alexa is very loose. We talk, that's it, that's my relationship, nothing personal. Comparable to the relationship to the Asian neighbor, who used to live in the basement. Once I saw him, I exchanged a few words with him, but he had no place in my heart. So, I didn't develop a personal relationship with Alexa." (P11, final interview)

Further, our results also showcase that some participants without initial privacy concerns formed a relationship with the smart speaker and humanized it. For instance, one participant described Alexa as "a fourth person in their apartment". Concurrently, they later became reluctant to discuss personal issues "in her presence". This indicates that the emerging relationship with the device can potentially lead to increasing privacy concerns as users begin to perceive the speaker as more human-like and thus a potential threat to privacy: "My relationship with Alexa is comparable to a human relationship, because, at our flat, Alexa is actually a person. [...] She's actually like a fourth person in the flat. [...] Yes, but I wouldn't discuss private information in front of her [i.e. Alexa]." (P32, mid-term interview)

Some users reported that their attitude towards the smart speaker changed in due course, while others emphasized that more time was needed to build a relationship with the device. The latter is illustrated by the following statement, where the user comments on the connection between the frequency of use, the time period of use and the relationship with the Echo: "I didn't develop a personal relationship with the echo. [...] We just had her for a limited period of 
time and I knew that she will be gone after that. Also, we did not use her that much, so no relationship could develop." (P21, final interview)

In contrast, other participants emphasized that interacting with the technology did not feel like interacting with an actual human being, even though some functionalities of the Echo were assessed very positively. One participant praised the voice of Alexa but also contemplated the reasons why he did not build a deeper relationship with her. He reflected that if Alexa became more human-like, the smart speaker would turn into a companion, comparable to a pet: "It's not a romantic relationship. I will not miss her when she's gone, even though it was nice. Alexa is not completely human-like yet, but it remains unclear why that is so. Therefore, I think it is difficult to really develop something that can be called a relationship. As I said, the voice is super pleasant and very human, but there is no character behind it, the consciousness, something human is missing. That's what the whole world is working on I suppose. [...] Then the feeling of 'I'm happy that she's here' is going to develop. People are buying pets to have company, and that's how it is going to be with smart speakers. For sure." (P72, final interview)

Furthermore, our data analysis indicates that the social setting influences differences in relationship formation. In multi-person households (e.g., families, shared apartments), the interaction with Alexa led to the implicit or explicit formation of rules. In households with similar roles (shared apartment), participants described playful power struggles. In contrast, in households with a more prevalent hierarchy (family), the individual interactions remained playful, whereas the power struggles changed into something more stressful. Therefore, a tense atmosphere developed within some households, intensified by the fact that different participants from shared households had differing views regarding the question of whether rules around the Echo have been set or not. This is showcased in the following statement from a participant who set usage rules during meals for the children. It happened that the smart speaker was sometimes turned off since the device still responded to requests by the children when they were supposed not to use it. However, the husband of the participant stated that they had not established any rules regarding Alexa: I: "Your husband told me that you haven't established any rules regarding Alexa?"

P: "I have established one rule. Alexa must not be addressed while we're having lunch and in the morning. Because, in the beginning, the first thing the kids did was, that they came in here and demanded their favorite songs in full volume. Since then, I have set the rule that there is no Alexa during breakfast and lunch. Sometimes this worked, sometimes it didn't." (P81, final interview)

\section{DISCUSSION}

The thematic analysis helped us build an understanding of how smart speakers can be integrated into households. Here, we summarize and interpret the findings of our study and identify ways forward for developing smart speakers.

\subsection{Supporting existing and new routines}

Our findings indicate that smart speakers should support routines and not necessarily simply offer functionality. For instance, one participant recounted that she was wishing her Alexa 'Good Morning' every day. Since wishing the smart speaker a good morning does not seem to serve a purpose or lead to an apparent benefit, such interactions with the smart speaker are currently not supported in the interaction process. Alexa is not going to say 'Good Morning' when not greeted and the smart speaker is also not going to address the fact that she forgot to wish her a good morning. Supporting such interactions might lead to more positive interactions and, consequently, deeper engagement.

Further, we observed that the introduction of a smart speaker to a household prompted experimentation and altering the home's digital landscape. As we observed in the CONCERNS AND NuISANCES and BUILDING RAPPORT themes, users creatively adapted the features of their homes and their smart speaker to match their daily routines and preferences. Future smart speakers can explore this opportunity and further empower users to make creative use of the resources the digital home infrastructure provides. Through this, smart speakers may become the mediator technology that lets users become bricoleurs [18] in their homes.

\subsection{Non-functional use}

Another way forward is to explore to successfully implement ludic elements [21] in the design of smart speakers. As shown in our results (cf. Establishing Habits), users interact with Alexa in ludic ways. Gaver remarked that ludic design should de-emphasize the pursuit of external goals and maintain openness and ambiguity while promoting curiosity, exploration, and reflection. Consequently, the ludic design emphasizes playful exploration. The question remains if it is possible to implement ludic elements in the design of such technologies successfully. At first glance, the rationale of ludic design to offer multiple meanings [47] contradicts the rationale of the focused and task-oriented processes the smart speakers offer. Offering the user multiple ways to perform a specific task and playful, ludic interactions and consequently exploring how this might affect user engagement emerges as a challenge for future work. Consequently, our work suggests that future designs for smart speakers should offer indirect, ornamental ways to achieve common tasks or even expand the range of non-functional features.

\subsection{Reflecting the social landscape}

Further, our results indicate that smart speaker interactions could benefit from explicitly supporting social interaction through audienceaware behaviors. The technology could detect the social situation, for instance, if there is a communication going on or if there are people present in the home, who are not members of the household and adjust the amount of interaction accordingly. Our results confirm that the social context often determines the form of the interaction with a digital assistant [29], even at home. This is in line with findings from Benlian et al. [2]. They showed that unintentional voice activation of smart speakers led to interpersonal conflicts. They conducted an experimental vignette study and an additional cross-sectional study. Their participants already had experience with smart speakers. In contrast, we conducted a longitudinal in the wild study with novel users (i.e. without prior experience with smart speakers), as recommended by Benlian et al. [2].

Moreover, our results show that a context of use where multiple people are around the device might lead to issues which affect the group. For instance, if multiple users want to issue commands 
at the same time, this can potentially lead to a power struggle, which can have a negative impact on the group dynamic as well as affect the user experience with the smart speaker negatively. Hence, considering the social implications of integrating into existing social dynamics or limiting interaction based on social usage rules are avenues to be explored. This shows that contextual sensing for smart speakers should go beyond location (as proposed by Sciuto et al. [46]) and also include sensing the current composition of the group around the device, much like in the case of tabletops [25].

Further, the qualitative data confirmed past reports that smart speakers became social agents in the household, becoming a part of human-to-human relationships. We found that users built relationships with the device, which was not necessarily connected to the functionality of the technology. Consequently, we believe that if a personal relationship is developed (and it seems like Alexa is designed to support that), the design should incorporate ways of limiting the negative consequences of the absence of the device, e.g., when it malfunctions. This conclusion is further strengthened by the fact that some participants tended to consciously keep the device at a distance (e.g comparing it to the 'Asian neighbor'). Thus, we hypothesise that there is inherent reluctance to bonding with the device. This assumption partly confirms findings from Kuzminykh et al. [23] and extends them to novel users. Kuzminykh et al. [23] conducted a cross-sectional qualitaitve inquiry with 20 participants about the antropomorphized perceptions of conversational agents such as Amazon Alexa. They showed that smart speakers were perceived on a continuum from somewhat more approachable to somewhat more distant and, in line with our results, the perception of the conversational agent was partly influenced by the (un)satisfied need to engage in an emotionally warm dialogue with the system.

On a similar note, some participants clearly stated that they were aware that this device is just a technology, and they anthropomorphized it to make it work the way they wanted it to work, but that they did not develop a relationship with it. In contrast, some results in our study suggest that a smart speaker has the potential to become a new companion for users [35], comparable to a pet, or a 2019 tamagochi. On a similar note, Gao et al. [19] showed that the Amazon Echo can be perceived as a friend, an assistant, a family member or a partner. Their findings are based on an analysis of user reviews from the Amazon Echo product page. Consequently, our findings partly extend the results from Gao et al. [19] to interactions between smart speakers and novel users (i.e. users without any prior experience with smart speakers). However, the somewhat provocative question remains: How can we design such technologies to account for this 'predisposition'? Is it even needed to improve the companion qualities of smart speakers or will they become like the new tamagochis in due course anyway? We hypothesize that there might be a threshold. If the technology is not interactive enough, this would lead to no companion qualities, and if the device is too interactive, it would potentially cause annoyance. Furthermore, if the smart speaker is as interactive as a human companion, this could lead to dangers from a mental health perspective, such as developing a dependence on technology. Consequently, if a smart speaker has the potential to become a companion comparable to a social robot [13] (as shown in the statements where users interacted with the tech for no particular reason: 'we just talk'), then other design principles, such as the principles applicable for social robots should be considered.

A broader question that emerges from our work is whether smart speaker systems should avoid building rapport altogether, thus avoiding becoming a social agent for collocated users [38]. Our observations do not fully align with research by Clark et al. [6] which suggested that no relationship beyond one a user would have with human strangers is required for conversational agents. If future smart speakers support more features beyond their core functionality (e.g., jokes), some users may develop dependence or be discriminated. This appears to be particularly relevant in light of results that showed that users without a technical background have a tendency for more anthropomorphism [27].

\subsection{Decline in usage}

Through our analysis, we identified various factors that determine why smart speakers are abandoned by new users of smart speakers after an initial testing and novelty period, such as privacy concerns, no added value, and functionality issues. These findings are partly in line with previous work (e.g. [28, 30, 49]).

Usually, smart speakers are introduced in a home environment that is already populated with various home appliances. Thus, users need to integrate smart speakers into their existing home ecologies or smart home infrastructures. While it is easy to create a smart home around a specific smart speaker system by extending the infrastructure, it is much more difficult to integrate a smart speaker in existing ecologies as not all existing appliances in the home might be compatible to the smart speaker system. Also, if users do not own other smart home products, e.g., smart lighting, or do not have access to third services, e.g., a streaming subscription, these circumstances restrict which functions of their smart speakers users can use as we observed in the ConCERnS AND Nuisances theme.

Hence, the benefit of smart speakers is limited since they are only immediately effective in a rare and very specific structural set-up. This confirms the results by Sciuto et al. [46] who analyzed history logs of smart speaker users and found that their users bought additional smart home products that are compatible to their smart speaker system to be able to use more of the functionality spectrum of their smart speakers. Further, this echoes the results by Tolmie et al. [50] that designers of future systems such as smart speakers need to take also existing devices in domestic environments into account, e.g., by enabling the support of more home appliances.

The patterns behind the decline in usage of a smart speaker that we observed are also different that those reported by Cho et al. [5]. We did not identify any signs of what they named 'disappointing exploration'. The users who abandoned the smart speaker in our study never established a bond with the device. We hypothesise that cultural context may plays a role in how usage patterns of smart speakers develop as Cho et al performed their work in South Korea. Future research should examine cross-cultural perceptions of smart speakers.

Furthermore, many appliances that are already available in the users' homes support functionalities similar to those provided by smart speakers. We observed in the Establishing HaBits theme that users were already accomplishing specific tasks using their existing home appliances and established routines for them; e.g., 
setting the alarm in the evening before going to bed. While it is easy for users to integrate a smart speaker into new practices, it is time-consuming to modify existing routines in order to integrate a smart speaker in the process. This is also a reason why users abandon smart speakers and continue using other appliances such as their traditional alarm clocks. Therefore, smart speakers could specifically inform the user how using the smart speaker instead of other appliances can make the process to accomplish a particular task more efficient. For instance, setting up a routine to inform the users in the morning about the weather conditions while they are preparing for the new day in the bathroom is much more timeefficient than checking the weather conditions manually on the phone.

Furthermore, we observed that current smart speakers fail to follow the organisational structures within a home and respect the current context of the users as we observed in the EsTABLISHING HABITS and BUILDING RAPPORT themes. This is related to the relationship between technology usage and space ownership that was addressed by Edwards and Grinter [16] (i.e., how different inhabitants of a home can watch their own programs on the TV). Smart speakers need to take established social conventions between the different inhabitants into account in order to avoid an occurring loss of autonomy, e.g., a loss of the autonomy between parents and their children. The opportunity to establish explicit rules [11] (i.e., no device usage by the children during meals or allowing specific actions only for defined users) might help to keep the user in control [48] and, therefore, dissolve some of the concerns that we identified (e.g., someone else adds items into a user's shopping cart) and support the social conventions within a home at the same time.

Further, we observed in the CONCERNS AND NuISANCES theme that especially those who were not digital natives, users with a technical indifference, and users without experience with voice assistant systems became frustrated by difficulties in interacting with a smart speaker. This confirms the results of Luger and Sellen [27] that users of conversational agents without computer-science knowledge were missing a mental model of how conversational agents work. Therefore, smart speakers should offer optional in-depth information for, especially users who are not experienced in the interaction with voice assistant systems or older adults that are less experienced in technology usage.

\subsection{Limitations}

Our study is prone to certain limitations. First, this study investigates a sample of novel users who received a smart speaker. Hence, our sample might be biased. Some of the participants would probably not buy a smart speaker themselves which could have influenced the lack of social bond that lead to the abandonment of the smart speaker. While we do believe that this is a limitation of this study, it also offers an opportunity, since previous studies mainly focused on power-users, enthusiasts, and users who already owned a smart speaker for a certain period. In contrast, we focused on novel users, comparable to users who would have received a smart speaker as a present.

Another limitation is the study duration. Since we specifically focus on the integration and potential (non-)use of smart speakers, a longer study period potentially could have led to further insights about the intricacies of this process. Even though our findings indicate that the time frame of our study was long enough to account for the declining novelty effect, future work should investigate potential critical factors for long-term engagement with the technology.

Furthermore, even though we are convinced that our conscious decision to conduct a qualitative inquiry with individual interviews, a different methodological approach (e.g. collecting quantitative measures or group sessions) potentially would have led to different results. Hence, one challenge that emerges for future research is the exploration of our findings through, for instance, a longer inquiry that combines quantitative methods with a qualitative inquiry.

Finally, in our study, we asked the participants to place the smart speaker at a shared location in their homes to ensure that all inhabitants had access to the deployed smart speaker. This is decision could have influenced how the speaker was used by the participants, especially with others present in the household [29]. However, current statistics to the placement of smart speakers show that smart speakers are mainly placed in the living room or the kitchen ${ }^{2}$. These locations were also favored by our participants.

\section{CONCLUSION}

To gain an understanding how users integrate smart speakers to their lives after smart speakers are introduced in their domestic environments and investigate the social implications of the smart speaker usage, we conducted a study where we deployed an Amazon Echo Dot in nine households with a total 20 household members. In our study, we focused on the qualitative experience of new smart speaker users from day one of their smart speaker usages. During the study, we conducted multiple interviews with the participants to be able to analyze how the participants experienced the integration of the smart speaker into their home and daily routines. We observed that half of the households reported that their use of the smart speaker steadily declined while other households anthropomorphize the Amazon Echo. Our analysis revealed four themes that characterize how smart speakers are introduced to home environments, the process of the (non-) adoption of the device and the social behaviors connected to the Echo Dot. Further, we presented insights for the design of future smart speakers that offer an improved experience when introduced to a household and better support social dynamics at home.

\section{ACKNOWLEDGMENTS}

We acknowledge the support of the Leibniz ScienceCampus Bremen Digital Public Health (lsc-diph.de), which is jointly funded by the Leibniz Association (W4/2018), the Federal State of Bremen and the Leibniz Institute for Prevention Research and Epidemiology-BIPS.

\section{REFERENCES}

[1] Erin Beneteau, Olivia Richards, Mingrui Zhang, Julie A Kientz, Jason C Yip, and Alexis Hiniker. 2019. Communication Breakdowns Between Families and Alexa. In Proceedings of the 2019 CHI Conference on Human Factors in Computing Systems-CHI, Vol. 19. 1-14.

\footnotetext{
${ }^{2}$ techcrunch.com, mycroft.ai/blog
} 
[2] Alexander Benlian, Johannes Klumpe, and Oliver Hinz. [n.d.]. Mitigating the intrusive effects of smart home assistants by using anthropomorphic design features: A multimethod investigation. Information Systems fournal n/a, n/a ([n.d.]). https://doi.org/10.1111/isj.12243 arXiv:https://onlinelibrary.wiley.com/doi/pdf/10.1111/isj.12243

[3] Frank Bentley, Chris Luvogt, Max Silverman, Rushani Wirasinghe, Brooke White, and Danielle Lottridge. 2018. Understanding the Long-Term Use of Smart Speaker Assistants. Proc. ACM Interact. Mob. Wearable Ubiquitous Technol. 2, 3, Article 91 (Sept. 2018), 24 pages. https://doi.org/10.1145/3264901

[4] Ann Blandford, Dominic Furniss, and Stephann Makri. 2016. Qualitative HCI research: Going behind the scenes. Vol. 9. Morgan \& Claypool Publishers. 1-115 pages.

[5] Minji Cho, Sang-su Lee, and Kun-Pyo Lee. 2019. Once a Kind Friend is Now a Thing: Understanding How Conversational Agents at Home Are Forgotten. In Proceedings of the 2019 on Designing Interactive Systems Conference (San Diego, CA, USA) (DIS '19). ACM, New York, NY, USA, 1557-1569. https://doi.org/10. 1145/3322276.3322332

[6] Leigh Clark, Nadia Pantidi, Orla Cooney, Philip Doyle, Diego Garaialde, Justin Edwards, Brendan Spillane, Christine Murad, Cosmin Munteanu, Vincent Wade, et al. 2019. What Makes a Good Conversation? Challenges in Designing Truly Conversational Agents. (2019).

[7] Phil Cohen, Adam Cheyer, Eric Horvitz, Rana El Kaliouby, and Steve Whittaker. 2016. On the future of personal assistants. In Proceedings of the $2016 \mathrm{CHI}$ Conference Extended Abstracts on Human Factors in Computing Systems. ACM, 1032-1037.

[8] Benjamin R Cowan, Nadia Pantidi, David Coyle, Kellie Morrissey, Peter Clarke, Sara Al-Shehri, David Earley, and Natasha Bandeira. 2017. What can i help you with?: infrequent users' experiences of intelligent personal assistants. In Proceedings of the 19th International Conference on Human-Computer Interaction with Mobile Devices and Services. ACM, 43.

[9] Benjamin R. Cowan, Nadia Pantidi, David Coyle, Kellie Morrissey, Peter Clarke, Sara Al-Shehri, David Earley, and Natasha Bandeira. 2017. "What Can I Help You with?": Infrequent Users' Experiences of Intelligent Personal Assistants. In Proceedings of the 19th International Conference on Human-Computer Interaction with Mobile Devices and Services (Vienna, Austria) (MobileHCI '17). ACM, New York, NY, USA, Article 43, 12 pages. https://doi.org/10.1145/3098279.3098539

[10] Andy Crabtree, Tom Rodden, Terry Hemmings, and Steve Benford. 2003. Finding a Place for UbiComp in the Home. In UbiComp 2003: Ubiquitous Computing, Anind K. Dey, Albrecht Schmidt, and Joseph F. McCarthy (Eds.). Springer Berlin Heidelberg, Berlin, Heidelberg, 208-226.

[11] Andy Crabtree, Tom Rodden, Peter Tolmie, Richard Mortier, Tom Lodge, Pat Brundell, and Nadia Pantidi. 2015. House Rules: The Collaborative Nature of Policy in Domestic Networks. Personal Ubiquitous Comput. 19, 1 (Jan. 2015), 203-215. https://doi.org/10.1007/s00779-014-0771-6

[12] Andy Crabtree and Peter Tolmie. 2016. A Day in the Life of Things in the Home In Proceedings of the 19th ACM Conference on Computer-Supported Cooperative Work \& Social Computing. ACM, 1738-1750.

[13] Maartje MA De Graaf and Somaya Ben Allouch. 2013. Exploring influencing variables for the acceptance of social robots. Robotics and Autonomous Systems 61, 12 (2013), 1476-1486.

[14] Barbara DiCicco-Bloom and Benjamin F Crabtree. 2006. The qualitative research interview. Medical education 40, 4 (2006), 314-321.

[15] Aarthi Easwara Moorthy and Kim-Phuong L. Vu. 2014. Voice Activated Personal Assistant: Acceptability of Use in the Public Space. In Human Interface and the Management of Information. Information and Knowledge in Applications and Services, Sakae Yamamoto (Ed.). Springer International Publishing, Cham, 324334.

[16] W Keith Edwards and Rebecca E Grinter. 2001. At home with ubiquitous computing: Seven challenges. In International conference on ubiquitous computing. Springer, 256-272.

[17] Nicholas Epley, Adam Waytz, and John T Cacioppo. 2007. On seeing human: a three-factor theory of anthropomorphism. Psychological review 114, 4 (2007), 864.

[18] Daniel Fällman. 2003. Design-oriented Human-computer Interaction. In Proceedings of the SIGCHI Conference on Human Factors in Computing Systems (Ft. Lauderdale, Florida, USA) (CHI '03). ACM, New York, NY, USA, 225-232. https://doi.org/10.1145/642611.642652

[19] Y. Gao, Z. Pan, H. Wang, and G. Chen. 2018. Alexa, My Love: Analyzing Reviews of Amazon Echo. In 2018 IEEE SmartWorld, Ubiquitous Intelligence Computing, Advanced Trusted Computing, Scalable Computing Communications, Cloud Big Data Computing, Internet of People and Smart City Innovation (SmartWorld/SCALCOM/UIC/ATC/CBDCom/IOP/SCI). 372-380.

[20] Radhika Garg and Jenna Kim. 2018. An Exploratory Study for Understanding Reasons of (Not-) Using Internet of Things. In Extended Abstracts of the $2018 \mathrm{CHI}$ Conference on Human Factors in Computing Systems. ACM, LBW024.

[21] William W. Gaver, John Bowers, Andrew Boucher, Hans Gellerson, Sarah Pennington, Albrecht Schmidt, Anthony Steed, Nicholas Villars, and Brendan Walker 2004. The Drift Table: Designing for Ludic Engagement. In CHI '04 Extended
Abstracts on Human Factors in Computing Systems (Vienna, Austria) (CHI EA '04). ACM, New York, NY, USA, 885-900. https://doi.org/10.1145/985921.985947

[22] Rebecca E. Grinter, W. Keith Edwards, Mark W. Newman, and Nicolas Ducheneaut. 2005. The Work to Make a Home Network Work. In ECSCW 2005, Hans Gellersen, Kjeld Schmidt, Michel Beaudouin-Lafon, and Wendy Mackay (Eds.). Springer Netherlands, Dordrecht, 469-488.

[23] Anastasia Kuzminykh, Jenny Sun, Nivetha Govindaraju, Jeff Avery, and Edward Lank. 2020. Genie in the Bottle: Anthropomorphized Perceptions of Conversational Agents. In Proceedings of the 2020 CHI Conference on Human Factors in Computing Systems (Honolulu, HI, USA) (CHI '20). Association for Computing Machinery, New York, NY, USA, 1-13. https://doi.org/10.1145/3313831.3376665

[24] Josephine Lau, Benjamin Zimmerman, and Florian Schaub. 2018. Alexa, Are You Listening?: Privacy Perceptions, Concerns and Privacy-seeking Behaviors with Smart Speakers. Proc. ACM Hum.-Comput. Interact. 2, CSCW, Article 102 (Nov. 2018), 31 pages. https://doi.org/10.1145/3274371

[25] Khanh-Duy Le, Mahsa Paknezhad, Paweł W. Woźniak, Maryam Azh, Gabrielè Kasparavičiūte, Morten Fjeld, Shengdong Zhao, and Michael S. Brown. 2016. Towards Leaning Aware Interaction with Multitouch Tabletops. In Proceedings of the 9th Nordic Conference on Human-Computer Interaction (Gothenburg, Sweden) (NordiCHI '16). ACM, New York, NY, USA, Article 4, 4 pages. https://doi.org/10. $1145 / 2971485.2971553$

[26] Irene Lopatovska and Harriet Williams. 2018. Personification of the Amazon Alexa: BFF or a Mindless Companion. In Proceedings of the 2018 Conference on Human Information Interaction \& Retrieval (New Brunswick, NJ, USA) (CHIIR '18). ACM, New York, NY, USA, 265-268. https://doi.org/10.1145/3176349.3176868

[27] Ewa Luger and Abigail Sellen. 2016. Like having a really bad PA: the gulf between user expectation and experience of conversational agents. In Proceedings of the 2016 CHI Conference on Human Factors in Computing Systems. ACM, 5286-5297.

[28] Lydia Manikonda, Aditya Deotale, and Subbarao Kambhampati. 2018. What's up with Privacy? User Preferences and Privacy Concerns in Intelligent Personal Assistants. In Proceedings of the 2018 AAAI/ACM Conference on AI, Ethics, and Society (New Orleans, LA, USA) (AIES '18). Association for Computing Machinery, New York, NY, USA, 229-235. https://doi.org/10.1145/3278721.3278773

[29] Aarthi Easwara Moorthy and Kim-Phuong L. Vu. 2015. Privacy Concerns for Use of Voice Activated Personal Assistant in the Public Space. International fournal of Human-Computer Interaction 31, 4 (2015), 307-335. https://doi.org/10.1080/ 10447318.2014.986642 arXiv:https://doi.org/10.1080/10447318.2014.986642

[30] Aarthi Easwara Moorthy and Kim-Phuong L. Vu. 2015. Privacy Concerns for Use of Voice Activated Personal Assistant in the Public Space. International fournal of Human-Computer Interaction 31, 4 (2015), 307-335. https://doi.org/10.1080/ 10447318.2014.986642 arXiv:https://doi.org/10.1080/10447318.2014.986642

[31] Kris Nagel, Cory D Kidd, Thomas O'Connell, Anind Dey, and Gregory D Abowd. 2001. The family intercom: Developing a context-aware audio communication system. In International Conference on Ubiquitous Computing. Springer, 176-183.

[32] Clifford Nass, Youngme Moon, Brian J Fogg, Byron Reeves, and Chris Dryer. 1995. Can computer personalities be human personalities? In Conference companion on Human factors in computing systems. ACM, 228-229.

[33] Clifford Nass, Jonathan Steuer, and Ellen R. Tauber. 1994. Computers Are Social Actors. In CHI '94 (Boston, Massachusetts, USA). ACM, New York, NY, USA, 72-78. https://doi.org/10.1145/191666.191703

[34] Jasmin Niess, Sarah Diefenbach, and Axel Platz. 2018. Moving beyond assistance: psychological qualities of digital companions. In Proceedings of the 10th Nordic Conference on Human-Computer Interaction. ACM, 916-921.

[35] Jasmin Niess and Paweł W Woźniak. 2020. Embracing Companion Technologies. arXiv preprint arXiv:2004.07198 (2020).

[36] William Odom, John Zimmerman, Jodi Forlizzi, Hajin Choi, Stephanie Meier, and Angela Park. 2012. Investigating the presence, form and behavior of virtual possessions in the context of a teen bedroom. In Proceedings of the SIGCHI Conference on Human Factors in Computing Systems. ACM, 327-336.

[37] Gerard Oleksik, David Frohlich, Lorna M Brown, and Abigail Sellen. 2008. Sonic interventions: understanding and extending the domestic soundscape. In Proceedings of the SIGCHI conference on Human Factors in computing systems. ACM, 1419-1428.

[38] Thomas Olsson, Pradthana Jarusriboonchai, Paweł Woźniak, Susanna Paasovaara, Kaisa Väänänen, and Andrés Lucero. 2020. Technologies for enhancing collocated social interaction: review of design solutions and approaches. Computer Supported Cooperative Work (CSCW) 29, 1 (2020), 29-83.

[39] Martin Porcheron, Joel E. Fischer, Stuart Reeves, and Sarah Sharples. 2018. Voice Interfaces in Everyday Life. In Proceedings of the 2018 CHI Conference on Human Factors in Computing Systems (Montreal QC, Canada) (CHI '18). ACM, New York, NY, USA, Article 640, 12 pages. https://doi.org/10.1145/3173574.3174214

[40] Martin Porcheron, Joel E. Fischer, and Sarah Sharples. 2017. "Do Animals Have Accents?": Talking with Agents in Multi-Party Conversation. In Proceedings of the 2017 ACM Conference on Computer Supported Cooperative Work and Social Computing (Portland, Oregon, USA) (CSCW '17). ACM, New York, NY, USA, 207-219. https://doi.org/10.1145/2998181.2998298

[41] Alisha Pradhan, Kanika Mehta, and Leah Findlater. 2018. "Accessibility Came by Accident": Use of Voice-Controlled Intelligent Personal Assistants by People 
with Disabilities. In Proceedings of the 2018 CHI Conference on Human Factors in Computing Systems (Montreal QC, Canada) (CHI '18). ACM, New York, NY, USA, Article 459, 13 pages. https://doi.org/10.1145/3173574.3174033

[42] Amanda Purington, Jessie G. Taft, Shruti Sannon, Natalya N. Bazarova, and Samuel Hardman Taylor. 2017. "Alexa is My New BFF": Social Roles, User Satisfaction, and Personification of the Amazon Echo. In Proceedings of the 2017 CHI Conference Extended Abstracts on Human Factors in Computing Systems (Denver, Colorado, USA) (CHI EA '17). ACM, New York, NY, USA, 2853-2859. https://doi.org/10.1145/3027063.3053246

[43] Aung Pyae and Tapani N. Joelsson. 2018. Investigating the Usability and User Experiences of Voice User Interface: A Case of Google Home Smart Speaker. In Proceedings of the 20th International Conference on Human-Computer Interaction with Mobile Devices and Services Adjunct (Barcelona, Spain) (MobileHCI '18). ACM, New York, NY, USA, 127-131. https://doi.org/10.1145/3236112.3236130

[44] Nithya Sambasivan, Leena Ventä, Jani Mäntyjärvi, Minna Isomursu, and Jonna Häkkilä. 2009. Rhythms of Non-Use of Device Ensembles. In CHI '09 Extended Abstracts on Human Factors in Computing Systems (Boston, MA, USA) (CHI EA '09). Association for Computing Machinery, New York, NY, USA, 4531-4536. https://doi.org/10.1145/1520340.1520695

[45] Christine Satchell and Paul Dourish. 2009. Beyond the User: Use and Non-Use in HCI. In Proceedings of the 21st Annual Conference of the Australian Computer Human Interaction Special Interest Group: Design: Open 24/7 (Melbourne, Australia)
(OZCHI '09). Association for Computing Machinery, New York, NY, USA, 9-16. https://doi.org/10.1145/1738826.1738829

[46] Alex Sciuto, Arnita Saini, Jodi Forlizzi, and Jason I. Hong. 2018. "Hey Alexa, What's Up?": A Mixed-Methods Studies of In-Home Conversational Agent Usage. In Proceedings of the 2018 Designing Interactive Systems Conference (Hong Kong, China) (DIS '18). ACM, New York, NY, USA, 857-868. https://doi.org/10.1145/ 3196709.3196772

[47] Phoebe Sengers and Bill Gaver. 2006. Staying open to interpretation: engaging multiple meanings in design and evaluation. In Proceedings of the 6th conference on Designing Interactive systems. ACM, 99-108.

[48] Ben Shneiderman. 2005. Shneiderman's eight golden rules of interface design. Retrieved fuly 25 (2005), 2009

[49] Madiha Tabassum, Tomasz Kosinski, and Heather Richter Lipford. 2019. "I don't own the data": End User Perceptions of Smart Home Device Data Practices and Risks. In Fifteenth Symposium on Usable Privacy and Security (SOUPS 2019). USENIX Association, Santa Clara, CA. https://www.usenix.org/conference/ soups2019/presentation/tabassum

[50] Peter Tolmie, Andy Crabtree, Tom Rodden, Chris Greenhalgh, and Steve Benford. 2007. Making the home network at home: Digital housekeeping. In ECSCW 2007, Liam J. Bannon, Ina Wagner, Carl Gutwin, Richard H. R. Harper, and Kjeld Schmidt (Eds.). Springer London, London, 331-350. 